Pacific Journal of Mathematics

Wetiaberpux Trenstrive Grour 


\title{
ON QUADRUPLY TRANSITIVE GROUPS
}

\author{
E. T. PARKER
}

1. Introduction. A major unsolved problem in the theory of groups is whether there are any quadruply transitive finite groups other than the alternating and symmetric groups and the four Mathieu groups of degrees $11,12,23$, and 24 , respectively.

In this paper are proved two theorems which impose arithmetic restrictions on primes dividing the order of the subgroup fixing four letters of a finite quadruply transitive group, and on the degrees of Sylow subgroups thereof.

THEOREM 1 is stated, followed by a corollary, which is somewhat less general but of a more direct arithmetic form.

THEOREM 1. If $G$ is a quadruply transitive finite permutation group, $H$ is the subgroup of $G$ fixing four letters, $P$ is a Sylow p-subgroup of $H, P$ fixes $r \geqq 12$ letters and the normalizer in $G$ of $P$ has component $A_{r}$ or $S_{r}$ permuting the letters fixed by $P$, and $P$ has no component of degree $\geqq p^{3}$ and no set of $r(r-1) / 2$ permutation-isomorphic components, then $G$ is alternating or symmetric.

COROLlary. If $G$ is a quadruply transitive permutation group of degree $n=k p+r$, with $p$ prime, $k<p^{2}, k<r(r-1) / 2, r \geqq 12$, and the subgroup of $G$ fixing four letters has a Sylow p-subgroup $P$ of degree $k p$, and the normalizer in $G$ of $P$ has component $A_{r}$ or $S_{r}$ permuting the letters fixed by $P$, then $G$ is $A_{n}$ or $S_{n}$.

This corollary is a partial generalization of a theorem of G. A. Miller [6], which may be paraphrased to read like the above with "quadruply transitive" replaced by "primitive" and the inequalities replaced by " $k<p, k<r, r \geqq 5$ " $-r \geqq 3$ " if the component of the normalizer of $P$ is restricted to be $S_{r}$. Miller's theorem is proved for $r \geqq 5$ by showing first that the component $A_{r}$ of the normalizer of $P-$ or a subgroup of $P$ of index 2 -splits off as a direct factor. The argument is completed by invoking the theorem of Netto [3, p. 207, Th. I] on primitive groups with primitive subgroups of lower degree. The proof of Theorem 1 makes use of the techniques in Miller's theorem; and in addition results on the structure of the automorphism groups of noncyclic groups of order $p^{2}$, on distribution of primes, and in particular

Received September 17, 1958. This paper is based on the author's dissertation, written at the Ohio State University in 1957, under the supervision of Professor Marshall Hall, Jr. The author expresses his gratitude to Professor Hall for guidance and encouragement. 
a theorem of Bochert [1] giving a lower bound on the degrees of permutations in quadruply transitive groups.

A consequence of Miller's theorem, not mentioned in his paper, is that for infinitely many degrees, namely $p+3$ and $2 p+3$ with $p$ prime, quadruply transitive groups can be only the alternating or symmetric groups.

THEOREM 2. If $G$ is a quadruply transitive permutation group of degree $n$, and the subgroup of $G$ fixing four letters is of order divisible by an odd prime $p$, with $5 p>n-4,4 p \neq n-4$, then $G$ is $A_{n}$ or $S_{n}$.

Some comments on terminology are in order: $A_{n}$ and $S_{n}$ designate respectively the alternating group and the symmetric group of degree $n$. A component of a permutation group is the permutation group induced on a transitive set of letters. "The subgroup fixing four letters" of a quadruply transitive group is the largest subgroup (unique to conjugacy) fixing four letters individually. (The phrase "fixed set-wise" is used explicitly when appropriate.)

Included in the author's dissertation is a self-contained proof that the only quadruply transitive groups of degrees $n \leqq 27$ are the alternating and symmetric groups, $6 \leqq n \leqq 27$ and $4 \leqq n \leqq 27$ respectively, and the Mathieu groups of degrees 11, 12, 23, and 24. As this result is in the literature (except perhaps for degree 27), though likely in no single source, these rather lengthy arguments are not included in this paper.

Section 2 contains three lemmas needed to prove Theorem 1 . In $\S \S 3$ and 4 are the proofs respectively of Theorem 1 and its corollary, and Theorem 2.

\section{In this section are three lemmas.}

LEMMA 1. If $B$ is a transitive permutation group of degree $p$ or $p^{2}$ ( $p$ prime), and if $B$ has a normal $p$-subgroup, then $B$ has no composition factor ${ }^{1}$ isomorphic with $A_{r}, r>5$.

Proof. If $B$ is of degree $p$, then $B$ is the metacyclic ${ }^{2}$ group or a subgroup thereof, and hence is solvable.

If $B$ is of degree $p^{2}$, then the normal $p$-subgroup $T$ has an elementary subgroup (not necessarily proper) $C$ normal in $B$. (For $p$-groups are solvable, and every minimal normal subgroup is a direct product of isomorphic simple groups.) Since $B$ is transitive, $C$ displaces all $p^{2}$

\footnotetext{
1 A factor group of a composition series.

2 The holomorph of the group of order $p$.
} 
letters, and has all components permutation-isomorphic. $C$ being abelian has all components regular. Thus $C$ is either the regular elementary group of order $p^{2}$, or a subdirect product of $p p$-cycles.

If $C$ is regular, then $B$ is in the holomorph of the elementary group of order $p^{2}$. The only unsolvable [3, pp. 428-34] composition factor of this holomorph is $L F(2, p)$. The smallest alternating group of order divisible by $p$ is $A_{p} . \quad L F(2, p)$ is isomorphic with no alternating group whenever $p>5$, since $p\left(p^{2}-1\right) / 2<p ! / 2$. The only unsolvable proper subgroup of $\operatorname{LF}(2, p)$ is isomorphic with $A_{5}-L F(2, p)$ is isomorphic with $A_{5}$ for $p=5$, and has such a subgroup for $p \equiv \pm 1(\bmod 5)[3$, pp. 440-50].

If $C$ is a subdirect product of $p p$-cycles, then each element of $B$ must permute the transitive sets of $C$ among themselves; these are sets of imprimitivity for $B$. Let $K$ be the largest subgroup of $B$ fixing the sets of imprimitivity; $K$ is a normal subgroup of $B . \quad B / K$ is a permutation group on the transitive sets of $K$. Since $T$ is transitive and $K$ is intransitive, $T$ is not a subgroup of $K$. Hence $B / K$ has a normal $p$ subgroup. $B / K$ of degree $p$ is in the metacyclic group, and is therefore solvable. $K$ is a subdirect product of metacyclic groups. Thus $B$ is solvable.

Lemma 2. For $r \geqq 9, A_{r}$ has no subgroup of index $t, r<t<r(r-1) / 2$.

Proof. Assume that $A_{r}$ has a subgroup $L$ of index $t$ ( $r, t$ as above).

If $L$ is intransitive, then $L$ is in the group $M_{i}$ of even permutations in the direct product of $S_{i}$ and $S_{r-i}, 0<i<r . \quad M_{i}$ is of index $\left(\begin{array}{l}r \\ i\end{array}\right)$ in $A_{r} . \quad\left(\begin{array}{l}r \\ i\end{array}\right) \geqq r(r-1) / 2$ unless $i=1$ or $r-1$. For $i=1$ or $r-1, M_{i}$ is $A_{r-1}$, of index $r$ which fails to satisfy the strict inequality. $A_{r-1}$ has no proper subgroup of index $<r-1$; hence $M_{i}$ has no proper subgroup of index $<r(r-1) / 2$ in $A_{r}$.

There remains for consideration the case of $L$ transitive.

Let $q$ be a prime in the range $r / 2<q \leqq r$. If $L$ is of order divisible by $q$, then an element of $L$ is a $q$-cycle, and $L$ is primitive $[4, \mathrm{p}$. 162, Exercise 8]. If further $q \leqq r-3$, then $L$ is $A_{r}$ [6]. Transitive $L$ fulfilling the assumption must be of index in $A_{r}$ divisible by each prime $q, r / 2<q \leqq r-3$.

A theorem on distribution of primes will now be used $[2,7]$ : If $x \geqq 25$, then there exists a prime $q$ such that $x<q<6 x / 5$. A computation shows that for all $r \geqq 50$ there exist primes $q_{1}, q_{2}, q_{3}$ satisfying $r / 2<q_{1}<q_{2}<q_{3} \leqq r-3$. The existence of a triple of primes for each $r$ in the range $20 \leqq r<50$ is verified by inspection. For any $r \geqq$ $20, q_{1} q_{2} q_{3}>(r / 2)^{3}>r(r-1) / 2$. 
Degrees $9 \leqq r \leqq 19$ remain to be considered. A primitive proper subgroup of $A_{r}$ has no element a $q$-cycle, with prime $q \leqq r-3$. If $L$ is primitive, then for each odd prime $q \leqq r-3$ a Sylow $q$-subgroup of $L$ must be a proper subgroup of that of $A_{r}$. Thus the index of $L$ in $A_{r}$ must be divisible by each prime $q, 2<q \leqq r-3$. This inequality is satisfied by primes $q=3,5,7,11$ when $14 \leqq r \leqq 19$; and $3 \cdot 5 \cdot 7 \cdot 11>$ $r(r-1) / 2$ for these values of $r$. Similarly $q=3,5,7$ for $10 \leqq r \leqq 13$, and $3 \cdot 5 \cdot 7>r(r-1) / 2$. Primitive $L$ does not exist for $10 \leqq r \leqq 19$.

Imprimitive $L$ must be of index divisible by each prime $q, r / 2<q \leqq$ $r$. For $17 \leqq r \leqq 19, q=11,13,17$, and $11 \cdot 13 \cdot 17>r(r-1) / 2$. For $13 \leqq r \leqq 16,11 \cdot 13>r(r-1) / 2$. For $r=11$ or $12,7 \cdot 11>r(r-1) / 2$. The maximal imprimitive subgroups of $A_{10}$ are of orders $\frac{1}{2} \cdot 2 !(5 !)^{2}$ and $\frac{1}{2} \cdot 5 !(2 !)^{5}$, both of index $>10 \cdot 9 / 2$. Such a subgroup of $A_{9}$ has order $\frac{1}{2} \cdot 3 !(3 !)^{3} ;$ index $>9 \cdot 8 / 2$.

One case remains, namely degree 9 with $L$ primitive. $L$ must be of index divisible by 3 and 5 . Since $3 \cdot 3 \cdot 5>9 \cdot 8 / 2, L$ can be of index only 15 or 30 in $A_{9}$. $L$, having a 7 -cycle, is triply transitive. If $L$ is of index 30 in $A_{9}$, then the subgroup of $L$ fixing two letters has order 84; a group of order 84 has only one Sylow 7 -subgroup. If $L$ is of index 15, then the largest subgroup of $L$ fixing two letters set-wise is of order 336 ; but a group of degree 7 and order divisible by $2^{4}$ contains a transposition.

Lemma 3. If $G$ is a transitive permutation group homomorphic onto $K$, and the kernel of the homomorphism is transitive on the letters permuted by $G$, then the subgroup of $G$ fixing one letter is homomorphic onto $K$. Moreover, the two homomorphisms belong to the same many-to-one mapping.

Proof. Let $G_{1}$ be the subgroup of $G$ fixing the letter 1 . For any $k \in K$, there exists $g_{k} \in G$ such that $g_{k} \rightarrow k$ in the homomorphism. Let $i$ be the letter onto which 1 is mapped by $g_{k}$. Being transitive, the kernel has an element $g_{k}^{\prime}$ mapping $i$ onto 1 . Then $g_{k} g_{k}^{\prime}$ maps 1 onto 1 , and corresponds to $k$ in the homomorphism. Since $k$ is an arbitrary element of $K$, it follows that $G_{1}$ has an element corresponding to any element of $K$.

3. In this section will be established.

THeOREM 1. If $G$ is a quadruply transitive finite permutation 
group, $H$ is the subgroup of $G$ fixing four letters, $P$ is a Sylow $p$-subgroup of $H, P$ fixes $r \geqq 12$ letters and the normalizer in $G$ of $P$ has component $A_{r}$ or $S_{r}$ permuting the letters fixed by $P$, and $P$ has no component of degree $\geqq p^{3}$ and no set of $r(r-1) / 2$ permutation-isomorphic components, then $G$ is alternating or symmetric

Proof. Let $N(P)$ be the normalizer in $G$ of $P$. Each element of $N(P)$ maps any component of $P$ onto a permutation-isomorphic component. Let $P^{\prime}$ be the largest subgroup of $N(P)$ fixing set-wise all components of $P . \quad P^{\prime}$ is a normal subgroup of $P$. The components of $P$ will be called points. $N^{*}=N(P) / P^{\prime}$ is a permutation group on points.

By hypothesis all points are of degrees $\leqq p^{2}$. By Lemma 1 no transitive group of degree $p$ or $p^{2}$ with a normal $p$-subgroup has a composition factor isomorphic with $A_{r}, r>5$. By hypothesis $N(P)$ has component $A_{r}$ or $S_{r}$ on the $r \geqq 12$ letters fixed by $P$. Thus $N(P)$, or a subgroup thereof of index 2 , is homomorphic with $A_{r}$. It follows that $N^{*}$ has a composition factor of $A_{r}$, since $P^{\prime}$ has none.

$N^{*}$ has a subgroup $N$ with properties:

1. $\quad N$ has an element permuting the $r$ letters fixed by $P$ according to $a$, where $a$ is any even permutation.

2. No proper subgroup of $N$ has property 1 .

A subgroup (not necessarily proper) of $N^{*}$ with property 1 exists, since $N^{*}$ itself has property $1 . N^{*}$, having only finitely many subgroups, has a minimal subgroup with property 1 . It is not asserted that $N$ is unique.

By the minimality condition on $N$, each component of $N$ is either a single point or is homomorphic with $A_{r}$, the image being represented on the letters fixed by $P$. Let $J$ designate a component of $N$ permuting more than one point (not letters fixed by $P$ ). $J$ is homomorphic with $A_{r}$, with kernel $J_{0}$ the subgroup of $J$ corresponding to the identity permutation of the letters fixed by $P$. Assume that the kernel $J_{0}$ is transitive on the points permuted by $J$. Then by Lemma 3 the subgroup of $J$ fixing one point possesses the homomorphism onto $A_{r}$; this contradicts the minimality property of $N$. Thus each $J$ has intransitive kernel.

For any $J$, the components of the kernel $J_{0}$ will be called blocks. Since $J_{0}$ is intransitive on the points permuted transitively by $J$, it follows that $J$ permutes more than one block. In fact, $J$ permutes blocks according to a group isomorphic with $A_{r}$, since $J_{0}$ is the kernel of the homomorphism of $J$ onto $A_{r}$. By hypothesis $P$ has no set of $r(r-1) / 2$ permutation-isomorphic components; thus each $J$ permutes fewer than $r(r-1) / 2$ points, and a fortiori fewer than this number of blocks. By 
Lemma $2 A_{r}$ for $r \geqq 9$ has no transitive permutation representation of degree strictly between $r$ and $r(r-1) / 2$ (by hypothesis $r \geqq 12$ ). Accordingly each $J$ permutes exactly $r$ blocks according to $A_{r}$.

For $r \neq 6$ the only automorphisms [3, p. 209] of $A_{r}$ are conjugations by elements of $S_{r}$. Thus there is a natural one-to-one correspondence between the letters fixed by $P$ and the blocks permuted by any $J$ such that each element of $N$ permutes the sets alike.

Select a set of $s=[r / 2]$ letters among the $r$ fixed by $P$. Let $N_{0}$ be a minimal subgroup of $N$ inducing all even permutations on these $s$ letters. (That $N_{0}$ exists is argued as for $N$.) As each $J$ is of degree $<r(r-1) / 2$, each block contains fewer than $(r-1) / 2$ points, hence fewer than $s$. The points of a block cannot be permuted according to any group homomorphic with $A_{s}$. By the minimality of $N_{0}$, each block fixed set-wise by $N_{0}$ is fixed point-wise by $N_{0}$. This is the case because $N_{0}$ has a composition factor of $A_{s}$, while the group permuting the points of one block has none.

$N_{0}$ is a group of permutations of points (transitive sets of $P^{\prime}$ ) and letters fixed by $P$. Thus $N_{0}$ determines a subgroup $M$ of $N(P)$ such that $N_{0}=M / P^{\prime} . \quad M$ permutes the chosen set of $s$ letters fixed by $P$ according to $A_{s}$. Let $M_{0}$ be a minimal subgroup of $M$ with this property. Each component of $P^{\prime}$ (point) is transitive of degree $p$ or $p^{2}$ and has a normal $p$-subgroup. Hence by Lemma 1, no component of $M_{0}$ containing a single component of $P^{\prime}$ has a composition factor of $A_{s}(s>5$, since $r \geqq 12$ ). Since $M_{0}$ is a minimal group homomorphic with $A_{s}$, each component of $P^{\prime}$ fixed set-wise by $M_{0}$ is fixed letter-wise by $M_{0}$. Thus each element of $M_{0}$ displaces at most $s / r \leqq 1 / 2$ of the letters of any component of $N(P)$; that is, at most half the letters displaced by $P$. $M_{0}$ has an element $m$ displacing exactly three letters fixed by $P$. As $r \geqq 12, m$ displaces at most half as many letters as the degree of $G$, diminished by 3 . The theorem of Bochert [1] asserts that quadruply transitive $G$, with a non-identical element displacing so few letters as does $m$, is alternating or symmetric.

COROLlaRY. If $G$ is a quadruply transitive permutation group of degree $n=k p+r$, with $p$ prime, $k<p^{2}, k<r(r-1) / 2, r \geqq 12$, and the subgroup of $G$ fixing four letters has a Sylow p-subgroup $P$ of degree $k p$, and the normalizer in $G$ of $P$ has component $A_{r}$ or $S_{r}$ permuting the letters fixed by $P$, then $G$ is $A_{n}$ or $S_{n}$.

Proof. Since $k<p^{2}, P$ is of degree $k p<p^{3}$, so that $P$ has no component of degree $\geqq p^{3}$. Since components of $P$ are of degree at least $p$, the hypothesis $k<r(r-1) / 2$ implies that $P$ has no set of $r(r-1) / 2$ permutation-isomorphic components. The hypothesis of Theorem 
1 is fulfilled, so that $G$ is $A_{n}$ or $S_{n}$.

4. THEOREM 2. If $G$ is a quadruply transitive permutation group of degree $n$, and the subgroup of $G$ fixing four letters is of order divisible by an odd prime $p$, with $5 p>n-4,4 p \neq n-4$, then $G$ is $A_{n}$ or $S_{n}$.

Proof. Let $H$ be the subgroup of $G$ fixing four letters, and $P$ be a Sylow $p$-subgroup of $H$. Since $5 p>n-4, P$ is of degree $k p \leqq 4 p$.

If $k p=n-4$, then $k \leqq 3$, since $4 p \neq n-4$ by hypothesis. The theorem of Miller [6] applies with $n=k p+4$ except for $k=p=3$. However, $13=2 \cdot 5+3$, so that Miller's theorem is applicable to this case.

If $k p<n-4$, then at least five letters are fixed by $P . N_{G}(P)$, the normalizer in $G$ of $P$, has component $T$ permuting the letters fixed by $P$ quadruply transitive [5, Lemma 2.2]. A normal subgroup (not the identity) of a quadruply transitive group (other than $S_{4}$ ) is triply transitive [3, p. 198, Th. XI]. Thus $T$ is primitive and has no regular normal subgroup, hence is unsolvable. The final subgroup in the commutator series of $T$ is triply transitive.

$P$ has at most four transitive sets, which cannot be permuted by an unsolvable group. If $p>3$, then each component of $P$ is of degree $p$ and has a solvable holomorph. For $p=3$, a component of degree 9 has a solvable automorphism group. Accordingly, the final member of the commutator series of $N_{G}(P)$ is a primitive group permuting only letters fixed by $P$. By Bochert's theorem [1], $P$ is of degree at least $(n-2) / 2$, so that at most $(n+2) / 2$ letters are fixed by $P$. Hence $G$ is at least $n+1-(n+2) / 2=n / 2-$ ply transitive [3, p. 207, Th. I.] But $G$ can be at most $(n+3) / 3-$ ply transitive, or contains $A_{n}$. [4, p. 148, Th. VI.] The theorem is established for $n>6$, and is trivial for the smaller degrees.

\section{REFERENCES}

1. Alfred Bochert, Ueber die Classe der Transitiven Substitutionen-gruppen, Math. Annalen 40 (1892), 176-193.

2. Robert Breusch, Zur Verallgemsinerung des Bertrandschen Posíulaies, dass zwischen $x$ und $2 x$ stets Primzahlen liegen, Math. Zeits., 34 (1932), 505-526.

3. W. Burnside, Theory of Groups of Finiie Order, 2nd ed., Cambridge Univ. Press, 1911; reprinted Dover, New York, 1955.

4. Robert D. Carmichael, Introduction to the Theory of Groups of Finite Order, Ginn, New York, 1937.

5. Marshall Hall, Jr., On a theorem of Jordan, Pacific J. Math. 4 (1954), 219-226.

6. G. A. Miller, Limits of the degree of transitivity of substitution groups, Bull. Amer. 
Math. Soc. 22 (1915), 68-71. See also Marshall Hall, Jr., The Theory of Groups, Macmillan, New York, 1959, pp. 68-72.

7. J. Nagura, On the interval containing at least one prime number, Proc. Japan Acad. 28 (1952), 177-181.

REMINGTON RAND UNIVAC

DIVISION OF SPERRY RAND CORPORATION

St. PAUl, Minnesota 


\section{PACIFIC JOURNAL OF MATHEMATICS}

\section{EDITORS}

\section{David Gilbarg}

Stanford University Stanford, California

\section{R. A. Beaumont}

University of Washington

Seattle 5, Washington

\section{A. L. Whiteman}

University of Southern California Los Angeles 7, California

L. J. Paige

University of California

Los Angeles 24, California

\section{ASSOCIATE EDITORS}

E. F. BECKENBACH
C. E. BURGESS
E. HEWITT
A. HORN

A. HORN

\author{
V. GANAPATHY IYER \\ R. D. JAMES \\ M. S. KNEBELMAN \\ L. NACHBIN
}

I. NIVEN

T. G. OSTROM

H. L. ROYDEN

M. M. SCHIFFER
E. G. STRAUS

G. SZEKERES

F. WOLF

K. YOSIDA

\section{SUPPORTING INSTITUTIONS}

\author{
UNIVERSITY OF BRITISH COLUMBIA \\ CALIFORNIA INSTITUTE OF TECHNOLOGY \\ UNIVERSITY OF CALIFORNIA \\ MONTANA STATE UNIVERSITY \\ UNIVERSITY OF NEVADA \\ OREGON STATE COLLEGE \\ UNIVERSITY OF OREGON \\ OSAKA UNIVERSITY \\ UNIVERSITY OF SOUTHERN CALIFORNIA
}

\author{
STANFORD UNIVERSITY \\ UNIVERSITY OF TOKYO \\ UNIVERSITY OF UTAH \\ WASHINGTON STATE COLLEGE \\ UNIVERSITY OF WASHINGTON \\ * * * * \\ AMERICAN MATHEMATICAL SOCIETY \\ CALIFORNIA RESEARCH CORPORATION \\ HUGHES AIRCRAFT COMPANY \\ SPACE TECHNOLOGY LABORATORIES
}

Mathematical papers intended for publication in the Pacific Journal of Mathematics should be typewritten (double spaced), and the author should keep a complete copy. Manuscripts may be sent to any one of the four editors. All other communications to the editors should be addressed to the managing editor, L. J. Paige at the University of California, Los Angeles 24, California.

50 reprints per author of each article are furnished free of charge; additional copies may be obtained at cost in multiples of 50 .

The Pacific Journal of Mathematics is published quarterly, in March, June, September, and December. The price per volume (4 numbers) is $\$ 12.00$; single issues, $\$ 3.50$. Back numbers are available. Special price to individual faculty members of supporting institutions and to individual members of the American Mathematical Society: $\$ 4.00$ per volume; single issues, $\$ 1.25$.

Subscriptions, orders for back numbers, and changes of address should be sent to Pacific Journal of Mathematics, 2120 Oxford Street, Berkeley 4, California.

Printed at Kokusai Bunken Insatsusha (International Academic Printing Co., Ltd.), No. 6, 2-chome, Fujimi-cho, Chiyoda-ku, Tokyo, Japan.

PUBLISHED BY PACIFIC IOURNAL OF MATHEMATICS, A NON-PROFIT CORPORATION

The Supporting Institutions listed above contribute to the cost of publication of this Journal, but they are not owners or publishers and have no responsibility for its content or policies. 


\section{Pacific Journal of Mathematics}

\section{Vol. 9, No. $3 \quad$ July, 1959}

Errett Albert Bishop, A minimal boundary for function algebras . . . . . . . . . . . . 629

John W. Brace, The topology of almost uniform convergence . . . . . . . . . . . . 643

Cecil Edmund Burgess, Chainable continua and indecomposability .......... 653

L. Carlitz, Multiplication formulas for products of Bernoulli and Euler

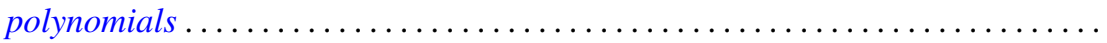

Eckford Cohen, A class of residue systems (mod $r$ ) and related arithmetical

functions. II. Higher dimensional analogues ....................

Shaul Foguel, Boolean algebras of projections of finite multiplicity . . . . . . . . . .

Richard Robinson Goldberg, Averages of Fourier coefficients .................

Seymour Goldberg, Ranges and inverses of perturbed linear operators .

Philip Hartman, On functions representable as a difference of convex functions ....

Milton Vernon Johns, Jr. and Ronald Pyke, On conditional expectation and

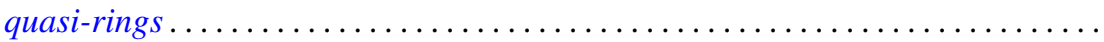

Robert Jacob Koch, Arcs in partially ordered spaces ....................

Gregers Louis Krabbe, A space of multipliers of type $L^{p}(-\infty, \infty) \ldots \ldots \ldots \ldots$

John W. Lamperti and Patrick Colonel Suppes, Chains of infinite order and their

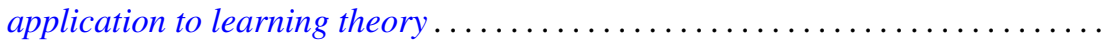

Edith Hirsch Luchins, On radicals and continuity of homomorphisms into Banach

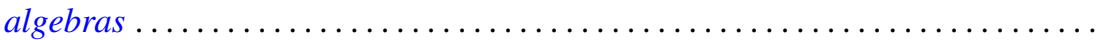

T. M. MacRobert, Multiplication formulae for the E-functions functions of their parameters.

Michael Bahir Maschler, Classes of minimal and representative domains and their kernel functions.

William Schumacher Massey, On the imbeddability of the real projective spaces in Euclidean space.

Thomas Wilson Mullikin, Semi-groups of class $\left(C_{0}\right)$ in $L_{p}$ determined by parabolic differential equations

Steven Orey, Recurrent Markov chains

Ernest Tilden Parker, On quadruply transitive groups ........ . .

Calvin R. Putnam, On Toeplitz matrices, absolute continuity, and unitary

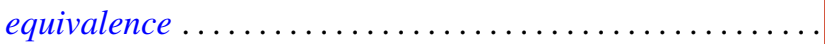

Helmut Heinrich Schaefer, On nonlinear positive operators.

Robert Seall and Marion Wetzel, Some connections between continued fractions and convex sets

Robert Steinberg, Variations on a theme of Chevalley

Olga Taussky and Hans Zassenhaus, On the similarity transformation between a

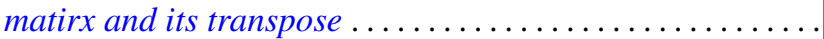

Emery Thomas, The suspension of the generalized Pontrjagin cohomology

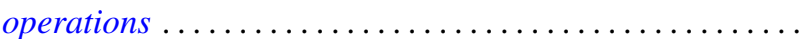

Joseph L. Ullman, On Tchebycheff polynomials ..................... 913

Richard Steven Varga, Orderings of the successive overrelaxation scheme ........ 925

Orlando Eugenio Villamayor, Sr., On weak dimension of algebras . 